\title{
Determination of quenching modes after thermodiffusion hardening of parts from gray cast iron
}

\author{
Vasiliev Valeriy Ivanovich, OvsiannikovViktor \\ Evgenievich \\ Kurgan State University \\ Kurgan, Russia \\ vik9800@mail.ru
}

\begin{abstract}
In this paper, the authors consider determination of the modes of heat treatment of gray cast iron parts that have been subjected to diffusion surface alloying. The type of hardening treatment under consideration makes it possible to obtain a layer of high-carbon steel on the surface of the cast iron part with the possibility of subsequent quenching on martensite. The application of the calculation method for determining quenching modes based on the solution of the Fourier equation in the criterial form is considered in the article.
\end{abstract}

Keywords— hardening, diffusion, cast iron, quenching

\section{INTRODUCTION}

The specificity of modern machine-building production is characterized by continuously increasing requirements for the quality of products and machines that are associated with increasing their productivity, accuracy and reliability. Numerous studies have established that the quality of machines is largely determined by the operational properties of parts and components that make up their composition. One of such properties is wear resistance in friction units.

The most important factors that determine the resistance of metals and alloys to wear include their structure, physical and mechanical properties, as well as their relative position, the quantitative ratio and the nature of the relationship between the individual components of the structure of the material. Proceeding from this, it can be concluded that the greatest durability should be the quality of a surface determined by a combination of the optimal structure and a complex of physicomechanical and physicochemical properties. However, it is practically impossible to obtain such complex of properties in practice, using a single-phase alloy.

It is known that in friction units, when an adjacent component is damaged, a layer of a certain thickness will almost always wear out, but most of the material of parts (often 90 or more percent) is not worn. Therefore, a promising way of ensuring the required wear resistance is surface hardening of parts made from a cheap material or the production of a composite material that has a complex of the properties listed above.

\author{
Nekrasov Roman Yurievich, Putilova Ulyana \\ Sergeevna \\ Industrial University of Tyumen \\ Tyumen, Russia \\ nekrasovrj@tyuiu.ru
}

Gray cast iron is a fairly common material for the manufacture of various parts of machines and structural elements. This material has a number of undeniable advantages, among which good casting properties, wear resistance (especially abrasive) and low cost are main.

However, one of the main drawbacks of this structural material is that it practically does not harden. The applied methods of hardening of this material [1-3] have low efficiency and require the use of expensive equipment and materials (laser installations, electrolytes, etc.).

With an increase in the eutectic degree of cast iron, the amount and size of graphite in its structure increase, the hardness of both the initial and hardened cast iron is significantly reduced.

Alloying elements slow down the diffusion of carbon in austenite, increase the hardenability of cast iron, and expand the range of cooling rates during quenching, which allows using softer quenching media (for example, oil instead of water) and thereby reduce the level of hardening stresses and deformations.

An increase in the austenitic temperature above A1 leads first to an increase in hardness due to a more complete austenitization, and then to a decrease in it, which is caused by supersaturation of austenite with carbon and an increase in the amount of residual austenite with an increase in the austenitization temperature from 1173 to $1223 \mathrm{~K}$. This causes a decrease in the hardness of cast iron by 20-30 HB.

If ferrite is present in the cast iron structure, the holding time for obtaining the maximum hardness increases, since dissolution of carbon in ferrite requires a longer time.

Induction surface hardening of cast iron is characterized by rapid heating and short-term aging at the austenitization temperature. In this case, the quenching capacity depends most strongly on the homogeneity of the initial cast iron structure, since a homogenization of austenite does not occur in a few seconds of heating for quenching. The presence of ferrite in the structure sharply worsens the quenching capacity. The quenching capacity is all the worse, the longer the holding time required to obtain the maximum hardness. Silicon promotes the formation of ferrite in the basis of gray cast iron. 
The chromium content of the base of gray cast iron significantly reduces the amount of structurally free ferrite and contributes to the production of high-dispersion perlite.

Alloying elements significantly increase the hardenability and quenching capacity of cast iron during induction hardening. However, with a large amount of ferrite in the initial microstructure, their effect is practically not manifested.

Improvement of gray cast iron consists in hardening with subsequent tempering. Depending on the tempering temperature, it is possible to obtain a troostite or sorbitol structure of the metal substrate, or a structure of granular perlite. Thus, improvement makes it possible to increase the hardness and strength of the original cast iron by obtaining structures, although less solid than martensitic, but harder than pearlitic or ferrite-pearlitic. The tempering temperature for improvement is selected depending on the requirements imposed on the structure and hardness of cast iron. The holding at the tempering temperature is selected from the condition of 1 hour for every $25 \mathrm{~mm}$ of the wall thickness.

The developed method of thermal diffusion hardening [4] makes it possible to obtain a layer of high-carbon steel on the surface of the cast iron product, with the simultaneous possibility of alloying with d-elements (chromium, titanium, molybdenum, etc.). The main advantages of this method are the increased thickness of the reinforced layer (up to $3 \mathrm{~mm}$ ), the absence of the need for complex equipment and the use of alloying elements as oxide materials, which additionally reduces the cost of the process.

However, in terms of practical applications, a problem arises in determining the modes of heat treatment (the heating time of parts). The main problem here is ensuring austenitic transformation, but exposure should be such that there is no growth of austenite grain.

\section{MEthod of CALCUlation OF THERMAL TREATMENT MODES}

The solution of the problem is to determine the dependence of temperature on the holding time in the furnace (construction of the heating curve). One of the most effective ways to determine this dependence is to solve the heat transfer equation [5].

The parameters for heating and cooling bodies depend on thermal conductivity $(\lambda)$ and thermal diffusivity $(\alpha)$.

Thermal conductivity depends on the composition of the material and on temperature [5]. With an increase in the carbon content and with the introduction of alloying elements, thermal conductivity decreases. As temperature rises, thermal conductivity decreases.

Thermal diffusivity is the ratio of thermal conductivity to the specific heat per unit volume of material [5]:

$$
a=\frac{\lambda}{c \gamma},
$$

where $c-$ heat capacity, $\lambda$ - coefficient of thermal conductivity and $\gamma-$ unit volume of material.
Heat transfer (a) is also an important factor, which is determined by the following factors [5]:

- nature of the heating or cooling medium;

- temperature of the medium;

- circulation of the medium;

- temperature of the heated or cooled surface of the product.

The heat transfer coefficient also depends on the shape and size of the product and the state of its surface. But the influence of the shape and size during heat treatment can be practically ignored, with the exception of heating and cooling of very small articles due to convection, and the influence of the state of the surface is comparatively small.

When parts are heated in a furnace, the total heat flux consists of the following parts:

- heat flux caused by radiation from the flame, combustion products, walls and roof of the furnace. The heat transfer coefficient corresponding to this component of the heat flux is denoted by $\alpha_{\text {rad; }}$;

- heat flux caused by convection from combustion products or air. The heat transfer coefficient due to this flux is denoted by $\alpha_{\text {conv }}$;

heat flux due to thermal conductivity from hearth or supports. Since this heat flux, as a rule, plays a very small role, it can be ignored.

The value of the convective part of the total heat transfer coefficient depends on the velocity of the gas flow and depends little on the temperature. The radiation-induced part of the heat transfer coefficient $\alpha_{\text {изл }}$ depends on the temperature of the heated body, roof, furnace walls and radiating gases.

The heat transfer coefficient can be calculated as the ratio of the heat flux through a unit of the body surface to the value of the thermal difference between the body surface and the medium [5]:

$$
\alpha_{\text {rad }}=\frac{q}{T-T_{\text {surf }}}
$$

where $\mathrm{q}$ - heat flux through a unit of the surface area; hearth;

$T$ - absolute temperature of radiating gases, vault and

$T_{\text {surf }}$ - absolute surface temperature.

Assuming some simplifications, one can write that in the case of heat transfer by radiation [5]:

$$
q=C\left[\left(\frac{T}{100}\right)^{4}-\left(\frac{T_{\text {surf }}}{100}\right)^{4}\right]
$$

where C - emissivity coefficient in $\mathrm{kcal} / \mathrm{m}^{2} \mathrm{hr} \mathrm{deg}^{4}$ for the oxidized surface of steel products, most often equal to about 4 . Hence [5]: 


$$
\alpha_{\text {rad }}=\frac{C\left[\left(\frac{T}{100}\right)^{4}-\left(\frac{T_{\text {surf }}}{100}\right)^{4}\right]}{T-T_{\text {surf }}},
$$

The parameters of the heat flux, taking into account the holding time of the object in the medium, can be determined by solving the Fourier equation [5]:

$$
\frac{\partial T}{\partial \tau}=\alpha \frac{\partial^{2} T}{\partial x^{2}}
$$

where $\mathrm{t}$ - temperature;

$$
\begin{aligned}
& \tau \text { - time; } \\
& \alpha \text { - thermal diffusivity; }
\end{aligned}
$$

However, it is more convenient to solve this equation in the criterial form [5]:

$$
\frac{T_{\text {mid }}-T}{T_{\text {mid }}-T_{0}}=F\left(\frac{x}{S} ; \frac{\alpha \tau}{S^{2}} ; \frac{\alpha S}{\lambda}\right),
$$

where a - coefficient of heat transfer from the surface of the body to the external medium;

$T_{\text {mid }}$ - temperature of the medium;

$T_{0}$ - initial body temperature;

$S$ - half the thickness of the plate, the radius of the cylinder or ball;

$\mathrm{x}$ - distance from the middle of the body to a given point;

$\mathrm{T}$ - current temperature at a given point;

$\lambda$ - coefficient of thermal conductivity;

$\alpha$ - coefficient of thermal diffusivity;

$\tau-$ time.

The quantities in the functional dependence are criterial functions:

$\frac{x}{S}$ - relative length that characterizes the position of the design section;

$F_{0}=\frac{\alpha \tau}{S^{2}}$ the process of heating (cooling);

$$
B i=\frac{a S}{\lambda}
$$
thermal resistance of the body to the intensity of heat exchange on the surface.

An important issue that needs to be addressed when calculating the heating and cooling of bodies is whether the body is thin or massive.

"Thin" bodies from the point of view of thermal calculations should be considered such bodies the temperature drop across the cross section of which is much less than the difference in the temperature of the surface of the product and the medium.

Therefore, a body belongs to the category of "thin" or "massive" depending on the ratio between the mass of the product and the intensity of supply or removal of heat from its surface. The less sharp the cooling or heating of the body, which is characterized by the coefficient of heat transfer a, the greater the cross section of the body that should be attributed to the category of "thin" by thermal calculations, and vice versa, the sharper the cooling or heating, the smaller the cross section of the product that should be attributed to the category of "massive".

This is due to the fact that with a sharper cooling, a certain temperature drop across the cross section of the product is obtained with a smaller cross section than in the case of less sharp cooling.

The ratio of the thermal drop within the product to the thermal difference between the product surface and the medium is determined based on the so-called Biot (Bi) criterion. This criterion is a ratio which, in the particular case of a plate, is equal to the ratio of the internal thermal resistance to the external thermal resistance at the metalmedium interface:

$$
B i=\frac{\frac{S}{\lambda}}{\frac{1}{\alpha}}=\frac{S \alpha}{\lambda}
$$

where $S / \lambda$ - internal resistance of the body;

$$
1 / \alpha \text { - external resistance. }
$$

The Biot criterion value is dimensionless.

To calculate the Biot criterion, the heat transfer coefficient when the body is heated or cooled is multiplied by $\mathrm{S}$, that is, by half the thickness of the plate or the radius of the cylinder or ball, and divided by the thermal conductivity of steel. In the case of plate cooling, only on one side, $\mathrm{S}$ is equal to the total thickness of the plate.

If the average value of the thermal conductivity coefficient is assumed to be constant $\left(25 \mathrm{kcal} / \mathrm{m}^{2} \mathrm{~h}^{\circ} \mathrm{C}\right)$, then for steel products, the Biot criterion can roughly be defined as $1 / 25$ of the product of $\mathrm{S}$ in meters by the heat transfer coefficient in $\mathrm{kcal} / \mathrm{m}^{2} \mathrm{~h}^{\circ} \mathrm{C}$.

If the Biot criterion differs little from 0 , then the cooling or heating of the body is determined only by the external heat removal, and the temperature distribution over the section does not play an important role, and the calculation can be carried out according to the formulas for thin products. Otherwise, cooling or heating should be calculated from the formulas for solid products, taking into account the thermal conductivity.

To establish the boundary between thin and massive products, it is necessary to set the degree of accuracy of calculations. The more accurate the calculations must be made, the narrower the area of thin articles, i.e., the smaller 
the cross-section of products that can be calculated by simplified methods that do not take into account the differences in temperature over their cross section. Excessive refinement of calculations is inexpedient, not only because of the laboriousness, but also because the initial data (heat transfer coefficients, thermal conductivity, etc.) are usually known only approximately, which makes calculation with higher accuracy superfluous. For our purposes it is possible to take the criterion of Biot number equal to 0.1 as the boundary value.

\section{Construction of the Theoretical Heating Curve}

The problem of constructing the heating curve consists in solving (2) for different values of the holding time in the medium $\tau$. The literature [5] shows the temperature dependence on the criterial functions. In this case, (2) is transformed in the following form:

$$
T_{i}=T_{\text {mid }}+\Theta_{S} \times \Theta_{R} \times\left(T_{0}-T_{\text {mid }}\right),
$$

where $\theta_{S}$ and $\theta_{R}$ - values of the criterial functions of the form $\theta(\mathrm{Bi}, \mathrm{Fo})$ for the surface and the middle of the sample, respectively.

As initial data for the calculation, the following values of the coefficients are taken[5]:

- coefficient of thermal conductivity $\lambda=14 \mathrm{kcal} / \mathrm{m} \cdot \mathrm{hr} \cdot{ }^{\circ} \mathrm{C}$;

- coefficient of thermal transfer $\mathrm{a}=150 \mathrm{kcal} / \mathrm{m}^{2} \cdot \mathrm{hr} \cdot{ }^{\circ} \mathrm{C}$ (corresponds to heating in an electric furnace);

- coefficient of thermal diffusivity $\alpha=0.025 \mathrm{~m}^{2} / \mathrm{hr}$.

${ }^{-}{ }_{\mathrm{Tmid}}=930^{\circ} \mathrm{C}$ (selected by recommendations [6]);

$-\mathrm{T}_{0}=25^{\circ} \mathrm{C}$.

The experimental sample is a cylinder with a diameter of $20 \mathrm{~mm}$ and a length of $50 \mathrm{~mm}$ (Fig. 1).

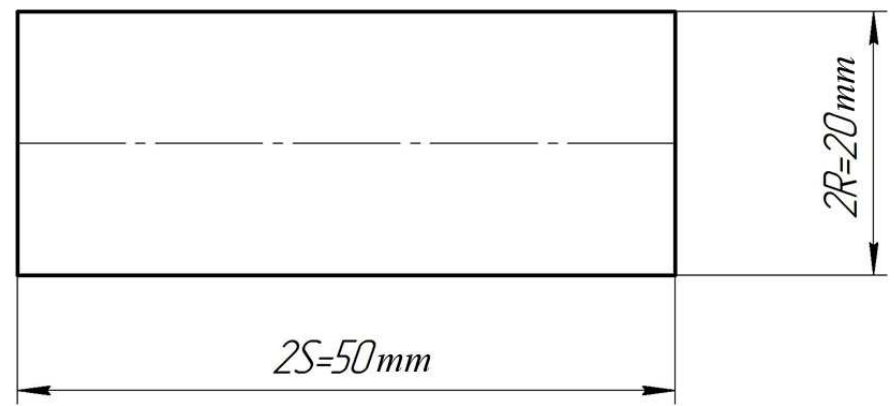

Fig. 1. Sample configuration

The values of the Biot criterion for the surface $S$ and the product axis $\mathrm{R}$ are respectively equal:

$$
\begin{aligned}
& B i_{R}=\frac{a R}{\lambda}=\frac{150 \cdot 0.5 \cdot 0.02}{14}=0.11 \\
& B i_{S}=\frac{a S}{\lambda}=\frac{150 \cdot 0.5 \cdot 0.05}{14}=0.26
\end{aligned}
$$

According to recommendations [4], from the abovedefined values of the Biot criterion, the sample under consideration can be referred to massive bodies. Therefore, determination of the values of the criterial functions will be carried out for such bodies.

The values of the Fourier criterion are determined depending on the holding time of the heated body in the medium. For convenience, the results of the calculations are summarized in a table.

TABLE I. VALUES OF THE FOURIER AND BIOT CRITERIA

\begin{tabular}{|l|l|l|l|l|}
\hline $\begin{array}{c}\text { Time } \\
(\boldsymbol{\tau}, \mathbf{h r})\end{array}$ & $\begin{array}{c}\text { Biot } \\
\text { criterion by } \\
\text { radius BiR }\end{array}$ & $\begin{array}{c}\text { Biot } \\
\text { criterion by } \\
\text { axis BiS }\end{array}$ & $\begin{array}{c}\text { Fourier } \\
\text { criterion by } \\
\text { radius FR }\end{array}$ & $\begin{array}{c}\text { Fourier } \\
\text { criterion by } \\
\text { axis FS }\end{array}$ \\
\hline 0.01 & 0.11 & 0.26 & 2.5 & 0.4 \\
\hline 0.03 & 0.11 & 0.26 & 7.5 & 1.2 \\
\hline 0.06 & 0.11 & 0.26 & 15 & 2.4 \\
\hline 0.08 & 0.11 & 0.26 & 20 & 3.2 \\
\hline
\end{tabular}

The values of the criterial functions as a function of the holding time in the medium are given in Table 2.

TABLE II. VALUES OF THE CRITERIAL FUNCTIONS

\begin{tabular}{|l|l|l|}
\hline Time $(\boldsymbol{\tau}, \mathbf{h r})$ & $\begin{array}{c}\text { Criterial function by } \\
\text { radius } \boldsymbol{\Theta}_{\mathbf{R}}\end{array}$ & \multicolumn{1}{|c|}{$\begin{array}{c}\text { Criterial function by } \\
\text { axis } \boldsymbol{\Theta}_{\mathbf{S}}\end{array}$} \\
\hline 0.01 & 0.64 & 0.75 \\
\hline 0.03 & 0.25 & 0.6 \\
\hline 0.06 & 0.03 & 0.35 \\
\hline 0.08 & 0 & 0.24 \\
\hline
\end{tabular}

Using the values of the criterial functions, we calculate the temperature of the heated sample at each specific time point, according to (3). The results of the calculations are shown in Table 3 and in Fig. 2.

TABlE III. PARAmETERS OF THE TheORETICAl HeAting CURVE

\begin{tabular}{|l|l|}
\hline \multicolumn{1}{|c|}{ Time $(\boldsymbol{\tau}, \mathbf{h r})$} & Sample temperature T, ${ }^{\mathbf{0}} \mathbf{C}$ \\
\hline 0.01 & 495 \\
\hline 0.03 & 794 \\
\hline 0.06 & 920 \\
\hline 0.08 & 930 \\
\hline
\end{tabular}

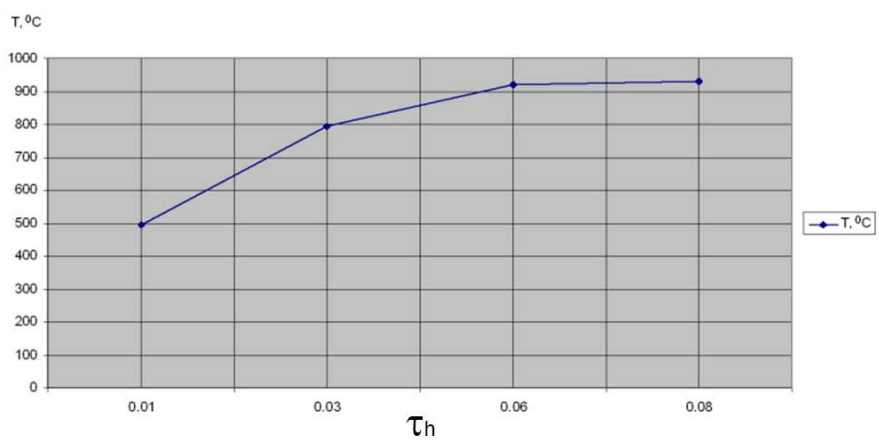

Fig. 2. Theoretical heating curve 
An important issue is checking the accuracy of the results of calculations. The inaccuracy of the above technique can be explained by the following reasons:

1) an inaccurate choice of initial values for calculating the thermal properties of the material, i.e., thermal conductivity and specific heat capacity, and the heat transfer coefficient. Errors in this case are the result of an incorrect choice of the average value of the thermal properties of the material and neglect of the dependence of the thermal properties of the material on temperature;

2) the error caused by mathematical reasons - the inaccuracy of tables and graphs for calculating the nonstationary thermal field, the inaccuracy of calculations by the finite difference method in the case of applying this method;

3) failure to take into account the thermal effects of structural transformations, for example, the transformation of austenite into perlite or martensite upon cooling, perlite or sorbitol into austenite upon heating and vice versa in the case of heating.

The specific heat capacity of steel changes in the range of the heat treatment temperature by approximately $\pm 20 \%$ of its average value. If the average value is correctly selected, the error in determining the heating or cooling time may be small. On the other hand, the calculation at a constant value of the specific heat capacity inevitably leads to some exaggeration of the heating and cooling time at room and low temperatures and to an understatement of this time at a high temperature.

The regularities of the change in the coefficient of thermal conductivity are exactly the same as for the specific heat capacity, so they should be considered in a single aspect.

The error caused by the inaccurate value of the heat transfer coefficient can be very different depending on a number of circumstances. For the calculation of the heating of massive bodies considered in the framework of this work, the most significant errors are in the calculation of the change in the surface temperature, since its rate of change is proportional to the heat transfer coefficient to the power of $n$, where $n$ lies between 1 and 2. The smallest error is obtained for the middle of the body, because for this part of the cross section the exponent lies between 1 and 0 . In the case of very massive bodies, for example, with $\mathrm{Bi}=10$ or more, the exponent for the surface is close to 2 , and for the middle to 0 . This means that a small error, for example, by $10 \%$ in the heat transfer coefficient, in this case leads to an error in calculating the rate of cooling or heating the surface by about $20 \%$ and for the middle of the body there will be almost no error.

The error, which is generated by the mathematical nature, is due to the fact that the values of the dependences of the criterial functions in different sources are approximated with different accuracy, which can reach the 5th decimal place. Therefore, an important point in this case is the choice of specific calculated curves to construct the desired heating (cooling) curve.

The influence of thermal effects that are generated by structural transformations in the material during heating (cooling) is characterized by the peculiarities of the course of heating (cooling) processes. At a usual slow heating with a furnace a considerable part of heat goes to warming up the furnace walls. During the perlitic transformation in steel, the temperature rise in it will cease, leading to a redistribution of heat, so that the rate of transformation will be higher than what follows from the formula. With further heating, the temperature rise will be slowed down due to the heat used for dissolving ferrite as a whole are not very significant and do not exceed $15 \%$.

All the above-mentioned circumstances give rise to the need for an experimental verification of the results of the calculated determination of the elements of the quenching regimes of the parts obtained above.

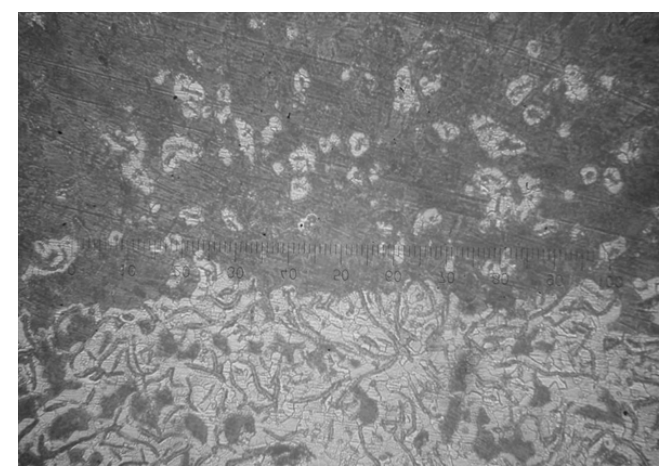

Fig. 3. Microstructure of the boundary of the hardened layer and the base of cast iron

\section{EXPERIMENTAL VERIFICATION OF THE RECEIVED DATA}

As can be seen from Table 3, the estimated heating and holding time in the furnace is approximately 5-6 minutes. Experimental verification of the accuracy of the obtained results and their applicability in practice was performed on a sample which geometric characteristic corresponds to Fig. 1.

The sample was heated in a furnace and held for 6 minutes, after which it was cooled in water. Fig. 3 shows the microstructure of the sample after this operation.

In this case, samples were selected which underwent thermal diffusion hardening by heating in a furnace in contact with titanium oxide at temperatures of $1000{ }^{\circ} \mathrm{C}$ and $1100{ }^{\circ} \mathrm{C}$ for 8 hours [4]. The initial structure of the sample after performing the process of thermal diffusion hardening but before quenching was pearlitic [4] (Fig. 3).

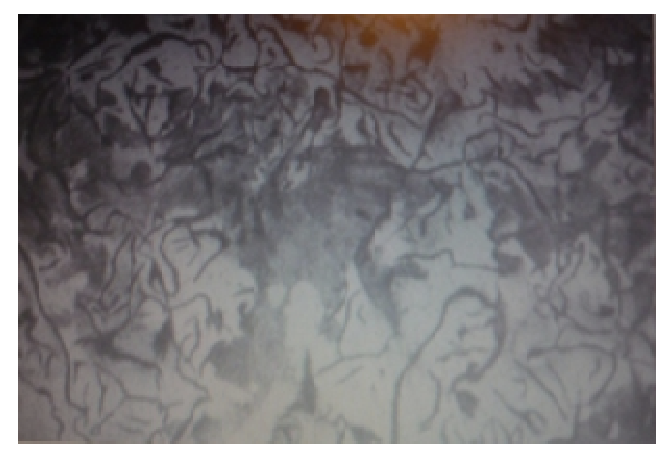

Fig. 4. Microstructure before quenching 


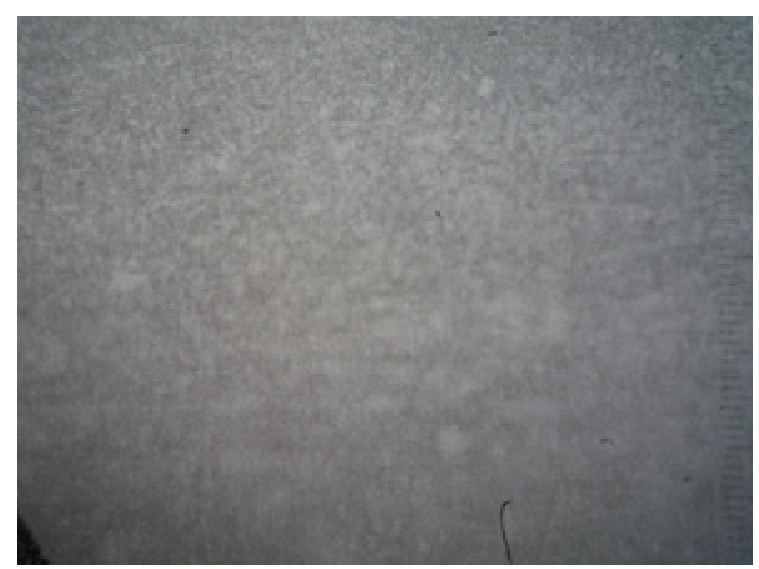

Fig. 5. Microstructure of the surface layer after thermal diffusion hardening (at $1000{ }^{\circ} \mathrm{C}$ ) and quenching

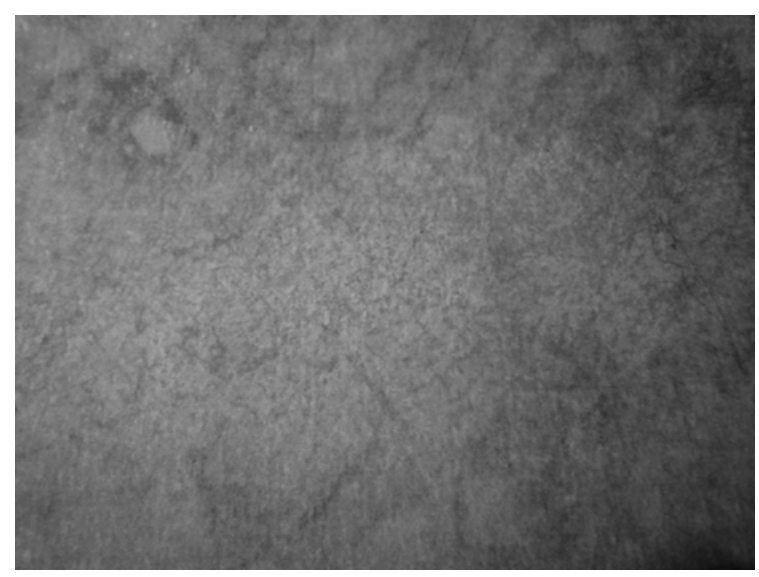

Fig. 6. Microstructure of the surface layer after thermal diffusion hardening (at $1100{ }^{\circ} \mathrm{C}$ ) and quenching

The distribution of microhardness over the thickness of the diffusion layer after the hardening process and subsequent quenching is given in Table 4 and in Fig. 6.

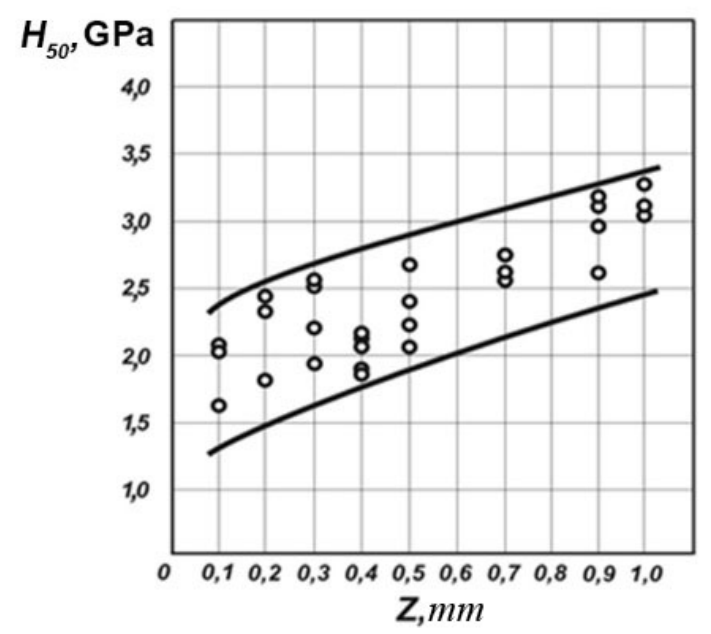

Fig. 7. Hardness distribution in the thickness of the layer after quenching
TABLE IV. DISTRIBUTION OF MICROHARDNESS OVER THE THICKNESS OF THE LAYER

\begin{tabular}{|l|l|l|}
\hline $\begin{array}{c}\text { Distance from the } \\
\text { sample surface, } \boldsymbol{\mu m}\end{array}$ & $\begin{array}{c}\text { Distance from the } \\
\text { sample surface, } \mathbf{~ m m}\end{array}$ & $H_{50}, \mathbf{G P a}$ \\
\hline 1191 & 1.191 & 2.86 \\
\hline 1357 & 1.357 & 2.32 \\
\hline 1523 & 1.523 & 2.10 \\
\hline 1689 & 1.689 & 1.75 \\
\hline 1855 & 1.855 & 1.61 \\
\hline 2021 & 2.021 & 1.37 \\
\hline
\end{tabular}

Fig. 4 and 5 show that the microstructure of the diffusion layer corresponds to fine-needled martensite. Thus, the calculated values of heat treatment modes have sufficient accuracy and can be used for practical purposes.

\section{References}

[1] V.S. Mayorov, "Quenching of cast iron parts by radiation of solid-state laser,” Metal Science and Heat Treatment of Metals, № 3, pp. 6-8, 2009.

[2] M.A. Krishtal, Mechanism of diffusion in iron alloys, Moscow: Metallurgy, 1972, 400 p.

[3] V.V. Povetkin, Structure of electrolytic coatings, Moscow: Metallurgy, $1989,136 \mathrm{p}$.

[4] Patent 2493289 Russian Federation, IPC C23C 10/36. Method of diffusion titanization of articles made of cast iron, Gurevich Yu.G., Ovsyannikov V.E., Frolov V.A., Sukhanov P.A.; the applicant and the patent holder is Kurgan State University. - №2012116651 / 02 applied 24/04/2012, published 09/20/2013, Bulletin No. 26.- 6 p .: il.

[5] A.L. Nemchinsky, Thermal calculations of heat treatment, Leningrad: Shipbuilding, 1953, $105 \mathrm{p}$.

[6] Yu.E. Sedov, Reference book of young thermist, Moscow: Higher Education, 1986, 239 p.

[7] U.S. Putilova, Yu.I. Nekrasov and A.A. Lasukov, Loading of the manufacturing systems elements in the process of unsteady mode cutting and the models of their arrangement deviations, Applied Mechanics and Materials, №682, pp. 192-195, 2014.

[8] R.Yu. Nekrasov, I.V. Soloviev and U.S. Putilova, Laser interferometry method of stress determination in loaded cutting tool parts, Applied Mechanics and Materials, №698, pp. 537- 541, 2015.

[9] R.Yu. Nekrasov, U.S. Putilova, A.I. Starikov and D.A. Kharitonov, Diagnostics of cut-layer deformation and rational tool loading in numerically contolled lathes, Russian engineering research, №34(12), pp. 826-828, 2014.

[10] R.Yu. Nekrasov, A.A. Starikov and M.A. Korchuganov Performance assessment of carbide tooling under thermal and loading conditions, Applied Mechanics and Materials, №682, pp. 414-417, 2014.

[11] A A Lasukov, P A Chazov, A V Barsuk Investigation on the Elemental Chip Formation Process in Hard-to-Machine Material Cutting Applied Mechanics and Materials, №682, pp. 504-509, 2014

[12] A A Lasukov Selection of machining conditions in terms of the temperature dependence of chip formation, Russian Engineering Research, 9, pp. 679-681, 2015. 